\title{
Médiation, éducation et formation
}

Jacky Beillerot

\section{(2) OpenEdition}

Journals

Édition électronique

URL : http://journals.openedition.org/trema/572

DOI : $10.4000 /$ trema. 572

ISSN : 2107-0997

Éditeur

Faculté d'Éducation de l'université de Montpellier

\section{Édition imprimée}

Date de publication : 1 mai 2004

Pagination : 27-34

ISSN : 1167-315X

\section{Référence électronique}

Jacky Beillerot, « Médiation, éducation et formation », Tréma [En ligne], 23 | 2004, mis en ligne le 06 janvier 2010, consulté le 01 mai 2019. URL : http://journals.openedition.org/trema/572 ; DOI :

10.4000/trema.572

Ce document a été généré automatiquement le 1 mai 2019.

Trema 


\title{
Médiation, éducation et formation
}

\author{
Jacky Beillerot
}

1 La notion de médiation en éducation est à la fois tellement évidente et polysémique qu'on est contraint de proposer une mise à plat de ses usages si l'on veut discuter les enjeux actuels qu'elle représente. L'effort de simplification est d'autant plus nécessaire que, sinon la médiation du moins les médiateurs, sont à la une de la presse tant le mot a pris de l'ampleur ces

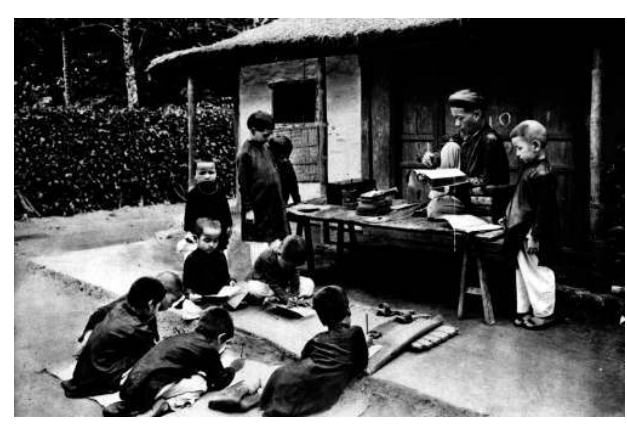
dernières années. C'est ainsi que les médiateurs se rencontrent dans les tribunaux, les immeubles, les quartiers, les entreprises, les banques, les hôpitaux, les diocèses, les journaux, et bien sûr dans l'Education nationale, administrations ou écoles ${ }^{1}$.

Assurer une médiation c'est jouer un rôle d'intermédiaire, celui d'un tiers plus ou moins reconnu comme neutre, en vue de créer ou de maintenir, entre des personnes, des groupes, des instances, des institutions, voire des Etats, des liens, qu'ils soient insuffisants ou inexistants ou qu'ils soient rompus.

3 La médiation ne date pas d'hier. Non seulement on la trouve sous différentes formes dans toutes les sociétés humaines, mais dans notre propre aire culturelle, elle puise ses origines à deux sources : la philosophie grecque et le christianisme. Les Grecs ont eu une vive conscience que le juge était un médiateur; Jésus est plusieurs fois nommé, dans le nouveau testament, comme le médiateur entre Dieu et les hommes (et les prophètes de l'ancien testament font aussi cet office) ${ }^{2}$. Ces rappels nous indiquent déjà que le médiateur occupe une position de milieu, où trois caractères, celui de la différence, de l'altérité et de la relation, lui permettent de jouer un rôle propre dans la conciliation de deux termes en état de division et d'opposition, pour faire surgir un résultat. 
4 La médiation doit d'abord être pensée dans le cadre du droit et de la justice. Elle fait même partie de ce qui se nomme, aujourd'hui, les «modes alternatifs de règlement des conflits » M.A.R.C ${ }^{3}$. Il s'agit de régler des différends autrement qu'en justice, il s'agit donc de processus non juridictionnels de traitement des litiges, une sorte de justice douce, à la confluence de la science du procès, de l'anthropologie juridique, des théories de l'information et de la négociation. Elle accompagne d'autres termes consubstantiels de la société moderne comme management, consensus, transparence, participation. Deux définitions précises cernent bien la nature de la médiation : «mode de construction et de gestion de la vie sociale grâce à l'entremise d'un tiers neutre, indépendant, sans autre pouvoir que l'autorité »; quant à l'autre définition elle voit la médiation comme "une méthode de résolution de litiges, basée sur un processus structuré, impliquant l'intervention d'une tierce personne et qui n'aboutit pas à une décision exécutoire imposée aux parties $»^{4}$. Dans les deux cas, c'est l'absence de pouvoir du médiateur qui est soulignée.

5 Une autre source juridique de la médiation doit au moins être rappelée : l'arbitrage et plus récemment l'arbitrage international. Si on constate que l'arbitrage a été utilisé depuis très longtemps, notamment, par les minorités, dans les sociétés à Etat faible ou naissant, au XIXe siècle, l'arbitrage comme pratique a reculé avec la montée de la puissance de l'Etat et des juridictions. En revanche, l'arbitrage explose au XXe siècle, aussi bien le commercial qui vise souvent la qualité des prestations nationales ou internationales dont on demande à des experts leurs jugements, que les rapports entre Etats. L'arbitrage est alors considéré comme une institution de paix.

6 Venons-en à la médiation et aux médiateurs dans le système scolaire et commençons par le plus récent ${ }^{5}$. Le 26 mai 2000, le médiateur de l'Education nationale remettait son premier rapport d'activité. Institué par un décret du premier décembre 1998, il est indépendant du médiateur de la République créé en 1973 (on se souvient que la Suède a inventé cette fonction au début du XXe siècle sous le nom d'OMBUDSMAN, celui qui est chargé de défendre les droits des citoyens face aux pouvoirs publics). Le médiateur de l'EN et les médiateurs académiques, chargés de prendre en considération les attentes individuelles des interlocuteurs, usagers et agents, ont eu à connaître lors de leur première année de travail, en 1999, 2400 réclamations, dont $73 \%$ proviennent des personnels, notamment du second degré, et qui concernent surtout des questions de carrière et de mutation. Globalement le résultat de la médiation a été favorable dans $63 \%$ des cas au demandeur, et défavorable dans $31 \%$ des dossiers. Dès cette première année, le médiateur recommandait à l'administration une approche plus qualitative de la gestion des personnels, une politique de responsabilisation individuelle des agents et demandait la création d'un comité de suivi des recommandations.

7 On peut maintenant se tourner vers d'autres médiateurs qui exercent dans l'école, ou plutôt examiner la diversité des fonctions de médiation avant de s'intéresser à des médiateurs stricto sensu' ${ }^{6}$.

8 La première fonction sert à interpréter l'activité de l'enseignant; celui - ci n'est plus seulement le dispensateur des connaissances mais agit pour être l'intermédiaire entre l'élève et les savoirs, construisant des situations et des dispositifs permettant à l'élève de surmonter les conflits psycho - socio - cognitifs propres à tout apprentissage ${ }^{7}$. 
Une deuxième fonction de médiation est explicite dans l'expression «remédiations cognitives » particulièrement employée dans le champ de la formation des adultes avant même d'entrer dans le système scolaire ${ }^{8}$. Il s'agit de programmes dont l'ambition est de "refonder" les apprentissages logiques de base par des progressions pédagogiques, à base d'exercices centrés sur l'entraînement au raisonnement ${ }^{9}$.

Plus récemment la médiation désigne la fonction assurée par certains personnels comme, les assistants sociaux, voire les inspecteurs, ou par certains enseignants comme les professeurs principaux, à qui expressément cette fonction est attribuée, ou encore les équipes éducatives mêmes de chaque établissement. La médiation vise ici aussi bien la vie à l'intérieur des classes et de l'école que les relations avec les familles et l'environnement.

Le quatrième emploi du terme de médiateur est réservé maintenant à de jeunes adultes mis à la disposition des établissements par le ministère, sous le terme générique "d'emploi - jeune », ou explicitement dénommés médiateurs éducatifs ou pédagogiques, et alors le plus souvent créés à l'initiative de collectivités territoriales. Leurs missions sont de prévenir ou de régler les conflits pour contribuer à l'apaisement des relations entre les jeunes, entre les jeunes et les adultes, missions pacificatrices et socialisantes en vue de permettre le déroulement normal des processus enseigner - apprendre ${ }^{10}$. On notera qu'en France, ces fonctions de médiateurs sont peu souvent attribuées à des élèves auprès de leurs pairs.

Les médiateurs en titre ou en sous - titre oserait-on dire, dans le système scolaire sont en partie associés à la réflexion sur la violence à l'école et font parfois partie de la panoplie des mesures visant à y remédier. C'est pourquoi des voix s'élèvent régulièrement contre les dérives possibles. Ainsi une principale de collège, Martine TAUSZIG raconte comment devant les difficultés de nombreux élèves d'une classe de $4 \mathrm{e}$, un espace de parole a été créé, animé par un conseiller d'orientation et un professeur chevronné volontaire ${ }^{11}$. Les heures « de vie de classe » avant la lettre fonctionnent bien. Mais rien de ce qui se passe dans ces moments ne transperce au niveau des équipes éducatives. Cet espace de parole est un lieu de régulation sans effet sur la classe. Les deux adultes sont devenus les confidents des élèves. «On a fabriqué de la parole contre et non de la parole avec ». Elle conclut «En fait, la classe semble être le premier lieu de médiation, le premier espace de parole organisé par l'enseignant... ». Et plus loin elle ajoute : «Les temps de délibérations et de négociations, c'est en fin de compte tout le temps $»^{12}$.

Pour être précis il faut, en cinquième lieu, examiner les fonctions de médiation de ceux qui sont appelés «les accompagnateurs scolaires ", vacataires salariés ou bénévoles que l'on connaît assez bien par divers travaux, synthétisés dans l'ouvrage de Dominique GLASMAN $^{13}$.Le chapitre 9 est consacré à notre thème, sous le titre "L'accompagnement scolaire artisan de rapprochement et scène de «médiation» entre Parents et Ecole? » (pp. 193 217). L'auteur entérine une distinction dans les fonctions des accompagnateurs, entre un rôle d'intermédiaire, de relais, qui vise à faire circuler des informations de l'école vers les parents et inversement, d'un rôle plus réellement de médiation, c'est - à - dire d'un tiers entre des parties. Mais être entre l'école et les familles soulève bien des problèmes. D'une 
part, l'institution scolaire s'estime beaucoup plus légitime à faire savoir aux parents, qu'à écouter voire apprendre quelque chose d'eux. Ensuite la position des accompagnateurs dans le critère de neutralité entre les « instances » semble bien variée selon les catégories sociales : les retraités cadres bénévoles sont plus enclins à tenter de faire comprendre les exigences de l'école que les accompagnateurs eux - mêmes issus des quartiers populaires dans lesquels ils exercent. Au final, on comprend bien qu'une vision politique globale sous - tend les manières de faire de la médiation : entend-on faire bouger l'institution scolaire et modifier le rapport qu'elle a avec les habitants, ou au contraire, estime - $t$ - on que les parents doivent s'adapter aux exigences de l'école? De même, plane le débat jamais bien réglé en France du droit à l'existence officielle des minorités et de la crainte de favoriser la constitution de catégories à part, de favoriser l'existence de communautés et de ce qui hante la philosophie politique, le communautarisme. Or certains médiateurs sur ce point peuvent être considérés comme suspects, voire comme agents doubles ${ }^{14}$.

C'est pourquoi, écrit D. GLASMAN, «On peut conjecturer que l'institution scolaire et les enseignants, sont en conséquence, davantage à la recherche de "relais» que de médiation. » (p. 210). Et l'école aurait alors tendance à demander, en fait, aux médiateurs d'être des vecteurs de normativité, de " considérer la citoyenneté (de certaines franges de la population?) davantage comme une acceptation et soumission à la norme commune que comme invitation à exprimer et faire valoir un point de vue critique. » (p. 211).

Ainsi la médiation et les médiateurs sont bien présents dans le système scolaire. Une présence marginale sans doute, y compris lorsque la médiation désigne le rôle central de l'enseignant comme intermédiaire entre les élèves et les savoirs, puisque cette représentation évidente pour certains, demeure très minoritaire.

Nous avons jusqu'alors peu parlé de la fonction de médiation en formation d'adultes, même si on a mentionné son existence dans les remédiations cognitives. C'est que l'affaire est tout à la fois plus simple et plus complexe. Plus simple, par exemple, car les formateurs sont plus enclins que les enseignants à se considérer comme des intermédiaires entre les savoirs des adultes et les « savoirs savants »; plus complexe car le champ de la formation des adultes est hétérogène, diversifié, et qu'il n'est pas régi par une institution unique sur le modèle séculaire d'un système scolaire. Plus complexe aussi, car les frontières sont plus floues : la présence des actions de médiations dans le champ du social, de la culture, de la justice, etc. sont toujours, à la fois spécifique et en même temps peuvent relever de la formation. La formation, en France, comprend en effet, toute l'activité de formation professionnelle, mais aussi de ce que nous appelons l'éducation populaire qui concerne la formation civile et civique, la formation culturelle ${ }^{15}$.

Il y a, au - delà de la variété des pratiques et des champs d'intervention de la médiation, plusieurs questions d'ordre philosophique et d'ordre anthropologique qu'on ne saurait trancher d'une manière abrupte.

$18 \mathrm{Au}$ sens propre, la médiation désigne une fonction «indirecte»; aux relations d'affrontement liées à une vision duelle et dichotomique de la réalité, la médiation offre, aussi bien dans le champ politique et social que dans celui de l'éducation, non seulement de nouveaux emplois, mais aussi la possibilité de relations plus élaborées et moins frontales, plus raisonnables, plus « rusées », donc moins brutales. 
19 La médiation contemporaine se caractérise aussi bien comme une intervention préventive de « déliaison » que comme une action en faveur de la résolution de conflits. Le travail du médiateur fait évoluer des règles et des comportements rigidifiés, entre deux parties ou entre un sujet et son environnement ; à cette fin, il implique un temps et un espace de transformation des représentations et des imaginaires jusqu'à une recomposition du champ symbolique des protagonistes, comme par exemple, apprendre à transiger.

Si un des enjeux des médiations est la déjudiciarisation des conflits, si d'autre part, il s'agit d'assurer des pilotages consensuels, visant à éviter, désamorcer, gérer des conflits (une justice alternative, plus humaine et moins coûteuse en somme), le médiateur est alors un rassembleur, un réconciliateur, un agent d'alliance, de communion, un modérateur, voire diront certains auteurs, un anesthésiste qui endort les populations puisqu'ils sont privés de tout pouvoir? Spécialiste de l'écoute, Antoine Adeline dit du médiateur qu'il est mi - prêtre, mi - psychanalyste. Espèce rare qu'il faut donc former, conclut-il!

Plus sérieusement si juger est dire le droit au nom du souverain, roi ou peuple, les M.A.R.C. remettent en cause l'antique modèle romain.

Se profileraient, alors, le mythe et l'illusion de la fin des conflits dont on sait que le risque demeure toujours celui d'une société totalitaire.

Jean - François SIX ${ }^{16}$ oppose la médiation - conflit celle qui vise à résoudre un conflit, à chercher des solutions, à une médiation - rencontre qui vise l'échange, la rencontre en elle - même. Il parvient alors à opposer les médiateurs qui sont dans un concept binaire et la médiation qui est radicalement ternaire, car une relation entre des individus établit des liens qui ont une consistance propre ; il s'agit moins alors d'une relation « à » que d'une relation " entre » des êtres. "Le "trois» est difficile; s'ouvrir à l'autre, à l'événement inattendu, est une tâche, celle de la médiation, une tâche toujours à recommencer, passionnante, ardue et qu'il s'agit de poursuivre tout au long de la vie.» (p. 439). Et J. - F. SIX conclut sa conférence en désignant la médiation comme une fraternité active qui requiert que l'on travaille d'abord à la liberté et à l'égalité. Citant LEVINAS « La vraie fraternité, c'est la fraternité par le fait que l'autre me concerne; c'est en tant qu'étranger qu'il est mon frère » il termine en écrivant "la médiation est entre nos mains » (p. 442).

Pour conclure à notre tour, revenons sur les ambivalences et les ambiguïtés de la médiation en général et sur la médiation à l'école.

D'une part, on peut distinguer une médiation qui prend la suite d'un conflit mené jusqu'à un certain moment dans un rapport de forces qui n'aboutit pas à déterminer un vainqueur et un vaincu; la poursuite des formes du conflit devenant trop coûteuses, les parties opposées cèdent, bon gré mal gré, et acceptent une médiation (exemples types, une grève, un conflit militaire). Le médiateur n'a pas le pouvoir de faire exécuter ses propositions, d'autres instances peuvent ou doivent s'en charger, à moins que les protagonistes ne s'y rallient « spontanément ».

D'autre part, la médiation vise à traiter des conflits, qui sans elle, soit s'enkystent comme dans les vendettas, soit relèvent des justices ordinaires. La médiation ici cherche à rapprocher les points de vue, à proposer des bénéfices à chacune des parties et la réussite 
se marque par l'accord des opposants. C'est la grande différence entre le travail de médiation et celui de la justice qui elle, vise, à dire le droit sans demander l'avis et encore moins l'accord des plaignants. La médiation a alors une fonction principale de prévention contre la détérioration des liens sociaux.

Enfin la médiation s'institutionnalise, dans les sociétés contemporaines sous deux grandes formes : les institutions se dotent de médiateurs dont les services sont offerts aux usagers ou clients et qui y ont recours s'ils le souhaitent. Mais le médiateur rémunéré par l'instance qui l'installe est donc sans réelle neutralité. Soit des parties opposées s'accordent pour demander à une tierce personne de leur choix de faire office de médiateur. Plus que dans tous les autres processus, un travail psychologique essentiel a été fait par les protagonistes, celui d'accepter de ne plus s'affronter, et celui d'avoir un premier accord en la personne du médiateur sollicité.

On voit donc bien comment le déploiement du phénomène de la médiation témoigne d'un changement en profondeur des rapports de pouvoir entre les instances, entre les individus ; moins de violence directe et davantage de négociations. Reste que la médiation pourrait ouvrir la porte à l'illusion et au mythe de la fin des conflits, ce qui à terme préparerait des lendemains désenchantés.

Cette complexité de la médiation ne peut pas ne pas être présente dans son exercice au sein de l'école. A la fois, elle 'assouplit» la vie quotidienne dans les établissements, elle tisse des relations entre école et environnement, mais en même temps, elle décharge la responsabilité des professionnels de l'école d'assurer eux - mêmes, la vie sociale de la classe et de l'établissement, ce qui n'est pas nécessairement un progrès.

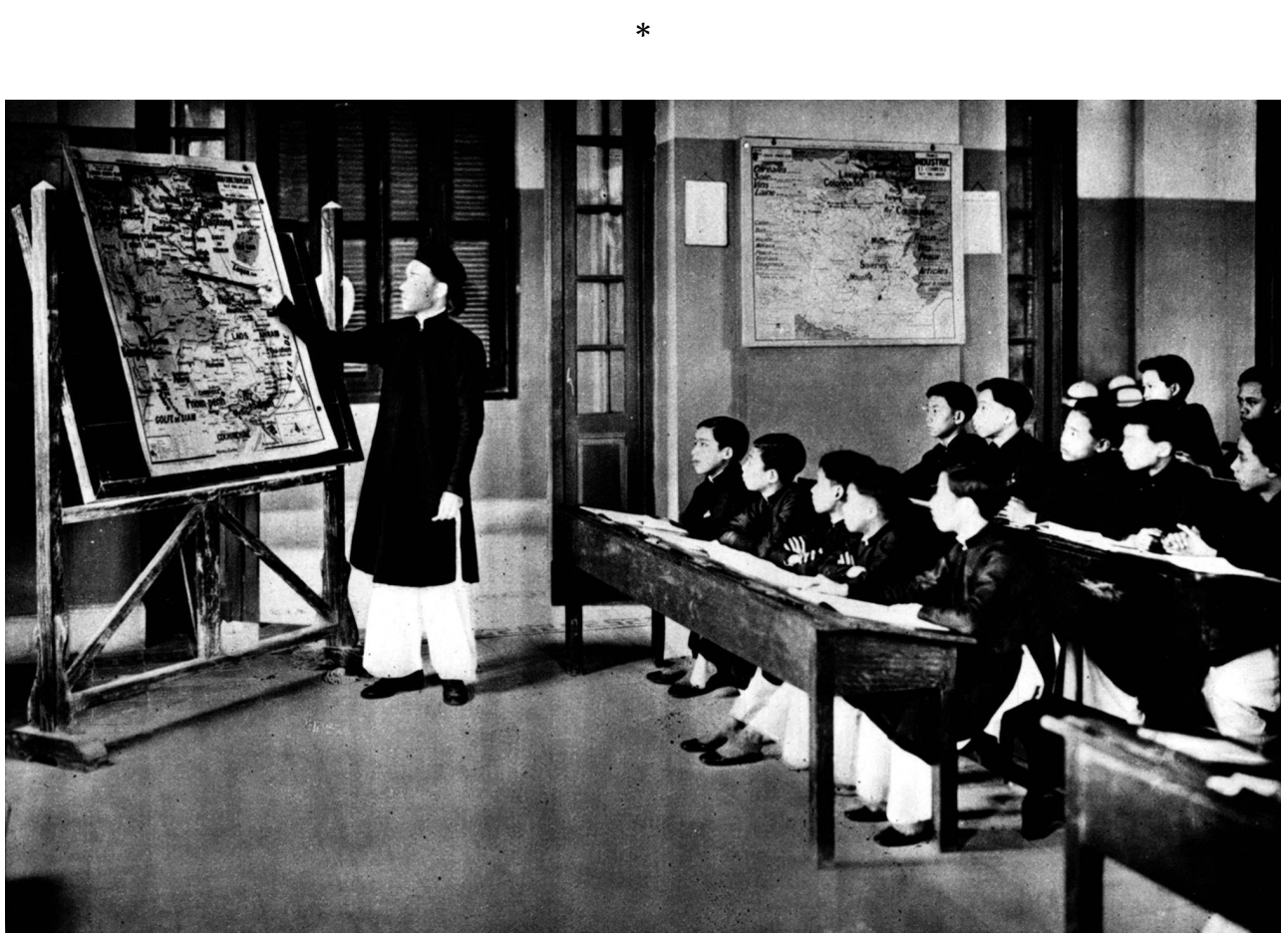

Une classe à l'école primaire d'Hanoï (Tonkin).

Image (légèrement réduite) tirée de : L'enfant colonial / Aimé Dupuy. Paris : Horizon de France, 1937. (Le visage de l'enfance). - p 280 


\section{BIBLIOGRAPHIE}

«La médiation : nouveau modèle de communication? (2002), L'école des parents. Hors série n 2, septembre.

ADELINE, Antoine, (1999), « Déjudiciariser les conflits : les enjeux de la médiation ». Universalia, pp 170 - 173.

CHARLOT, Bernard, (2002), "Aides - éducateurs : une dérive communautariste ? ", Cahiers

pédagogiques, n409, décembre, pp. 50 - 51.

GLASMAN, Dominique, (2001), « L'accompagnement scolaire. Sociologie d'une marge de l'école ». Paris : PUF. - La Lettre de l'éducation. 29 mai 2000. N 307, p. 1.

LARTIGOT, Jean - Claude, (1997), « La médiation dans les situations d'apprentissage d'un instrument de musique ", Les sciences de l'éducation pour l'ère nouvelle, vol. 30, n 3, pp. 85 - 114.

PRAIRAT, Eirick, (2001), Sanction et socialisation. Paris : PUF.

PRAIRAT, Eirick, (2002), « La lente désacralisation de l'ordre scolaire », Esprit, n¹2, pp. 138 - 151.

SIX, Jean - François, (2000), « La médiation. Des médiateurs ». Texte de la 127e conférence de l'Université de tous les savoirs, in Yves MICHAUD (dir.), «Qu'est - ce que la société ?» (pp. 432 442), Université de tous les savoirs, vol. 3. Paris : Editions Odile Jacob.

TAUSZIG, Martine, (1999), « Médiateurs, attention aux dérives », Cahiers pédagogiques, $\mathrm{n}^{\circ} 375$, juin, pp. 50 - 51.

ZAKHARTCHOUK, Jean - Michel, (1999), L'enseignant, un passeur culturel. Paris : ESF.

\section{NOTES}

1. «La médiation: nouveau modèle de communication?» L'école des parents. Hors série $n^{\circ} 2$. Septembre 2002. $65 \mathrm{p}$.

2. On peut aussi mentionner la création au début du XIXe siècle des prud'hommes et des juges de paix.

3. Cf. Antoine Adeline, 1999, «Déjudiciariser les conflits : les enjeux de la médiation». Universalia, pp. 170 - 173.

4. Ces deux définitions sont rapportées par ADELINE. La première est de Michèle GUILLAUME HOFNUNG, l'autre est due à MACKIE, MILES et MARCH.

5. La Lettre de l'éducation, $\mathrm{n}^{\circ} 307,29$ mai 2000, p. 1.

6. Je reprends dans ces lignes les éléments que j'avais développés dans l'article « Médiation» du Dictionnaire encyclopédique de l'éducation et de la formation (Nathan, 1998, 2e édition).

7. Le livre de Jean - Michel ZAKHARTCHOUK exprime très bien cette fonction, dans son titre d'abord, dans les développements du texte ensuite (L'enseignant, un passeur culturel. 1999, ESF, 124 p.). On peut encore rapporter l'étude de Jean - Claude LARTIGOT, «La médiation dans les situations d'apprentissage d'un instrument de musique (in Les sciences de l'éducation pour l'ère nou velle, 1997, n 3, vol. 30, pp. 85 - 114). L'auteur écrit «... le professeur se met en jeu dans la production de l'élève en lui permettant de faire quelque chose auquel il n'aurait jamais pu accéder seul dans le rapport avec l'instrument et la partition » (p. 85). 
8. Sans doute la remédiation n'est pas seulement une deuxième médiation entre l'individu et les savoirs ; elle est aussi une médiation qui vise à réparer les ratés de la première, une médiation de la deuxième chance. Remédier veut aussi dire porter remède.

9. A titre d'exemple, des chercheurs développent aujourd'hui des méthodes de remédiation de la lecture (lutte contre la dyslexie) sous forme de logiciels de plus en plus perfectionnés.

10. Les résultats d'une grande enquête conduite par la DPD et le CEREQ peuvent nuancer l'importance de la médiation; en effet, les fonctions les plus souvent confiées aux aides éducateurs sont relatives à la sécurité, à l'appui à l'enseignement, à la documentation et aux technologies de l'information et de la communication. La médiation, définie d'ailleurs comme «médiation auprès des familles et des centres sociaux » représente moins de $10 \%$ des activités, alors que les premières fonctions ont des scores allant de 60 à $80 \%$ (note d'information 02.15 mars 2001). Par ailleurs, le CEREQ propose huit profils - types des activités multiples de ces emplois - jeunes; deux nomment la médiation: animateur médiateur scolaire et médiateur scolaire.

11. « Médiateurs, attention aux dérives ». In Cahiers pédagogiques, juin 1999, n³75, pp. 50 - 51.

12. On peut aussi lire l'article de Eirick PRAIRAT, «La lente désacralisation de l'ordre scolaire » ( Esprit, 2002, n 12, pp. 138 - 151) qui suggère, à la suite de son livre Sanction et socialisation (2001, PUF) une autre voie : inventer une nouvelle ritualité de passage, argumentant que «l'école ne peut pas être le lieu vide des apprentissages, elle a, par sa nature / "d'entre - deux», un travail d'accompagnement et d'étayage à conduire» (pp. 10 - 151). Et il termine son article en écrivant : «Le rituel est en quelque sorte un micro culte rendu à ces idées qui comptent. Deux valeurs pourraient aujourd'hui guider notre action éducative : le respect et l'hospitalité. »

13. L'accompagnement scolaire. Sociologie d'une marge de l'école. 2001, PUF, 317 p.

14. Ce paragraphe était à peine écrit que sortait le numéro 409 des Cahiers pédagogiques (décembre 2002) qui comprend une interview de Bernard CHARLOT, à propos de son dernier ouvrage. Titre de l'article : «Aides - éducateurs : une dérive communautariste ? (pp. 50 - 51).

15. On peut ajouter que commencent à s'installer des formations spécifiques à la médiation et les premiers diplômes ont fait leur apparition.

16. «La médiation. Des médiateurs». Texte de la 127e conférence de l'Université de tous les savoirs, donnée le 6 mai 2000. In Yves MICHAUD (dir.), «Qu'est - ce que la société ?» (pp. 432 442), Université de tous les savoirs, vol. 3. Paris, Editions Odile JACOB, 897 pages.

\section{RÉSUMÉS}

La médiation existe depuis fort longtemps dans nombre de sociétés humaines et doit être étudiée d'abord dans le cadre du droit et de la justice. Dans le système scolaire la notion et la fonction de médiation ont diverses places; du Médiateur aux médiateurs éducatifs, la variété d'usage ne manque pas. En formation d'adultes, la médiation sans doute très présente est moins repérable qu'à l'école. Au-delà des situations d'apprentissage, l'essor de la médiation dans nos sociétés vise aussi bien une intervention préventive de "déliaison » qu'une action en faveur de la résolution de conflits. La médiation témoigne d'un changement des rapports de pouvoir entre les instances et entre les individus, impliquant des relations moins violentes et davantage négociées, ce qui ne doit pas conduire à l'illusion et au mythe de la fin des conflits. 
Mediation exists for a long time in many human societies and it must be studied first within the framework of law and justice. In the educational system, mediation as a notion and a function covers many places ; from the Mediator to the educational mediators, uses are legion. Mediation in training is probably really present but it's more difficult to spot than at school. Beyond the learning situations, mediation's development in our societies aims at preventive intervention of "deliaison" as well as action to promote the resolution of conflicts. Mediation shows a change in the balance of power between authorities and the individuals, whose relations are less violent and more negotiated. Even if this mustn't lead to illusion and myth of the end of the conflicts.

\section{INDEX}

Keywords : conflict, mediation, negociation, remediation

Mots-clés : conflit, médiation, négociation, rémédiation

\section{AUTEUR}

JACKY BEILLEROT

Professeur émérite, Université de Paris X, Nanterre 